\title{
Study on the Effect of the Thiourea on Nano-Mechanical Properties and Microstructures of the Electroformed Thin Ni-P Foil
}

\author{
Moo Young Jung ${ }^{1}$, Chang Su Nam ${ }^{1}$, Byung-Sik Lee ${ }^{2}$, and Yong Choi ${ }^{1, *}$ \\ ${ }^{l}$ Department of Materials Science and Engineering Dankook University, Cheonan 31116, Republic of Korea \\ ${ }^{2}$ Department of Nuclear Engineering, Dankook University, Cheonan 31116, Republic of Korea
}

\begin{abstract}
The effect of thiourea on the nano-mechanical and chemical properties of Ni-P foil was studied to develop a high performance Ni-P deposit for automobile and machinery parts. The Ni-P deposit was prepared by electroforming in a modified sulphate bath with different amounts of thiourea. Chemical analysis of the electrodeposited Ni-P foils by energy dispersive spectroscopy showed that phosphorous in the foil was reduced with thiourea. Structural analysis by X-ray diffractometry revealed that the (111) plane of the Ni-P deposits grew preferentially with increasing crystallinity. Surface analysis by atomic force microscopy and fieldemission scanning electron microscopy showed that the surfaces of the Ni-P electrodeposited with 0 and 0.01 $\mathrm{g} / \mathrm{L}$ thiourea were relatively smooth and clean showing no nodules, whereas, the cauliflower-like nodules were observed on the surfaces of the deposits prepared with $0.03,0.05,0.07$ and $0.1 \mathrm{~g} / \mathrm{L}$ thiourea. The surface roughness $\left(\mathrm{R}_{\mathrm{RMS}}\right)$ of the deposits increased by addition of $0.1 \mathrm{ppm}$ of thiourea from 6 to $54 \mathrm{~nm}$. Nanomechanical properties of the Ni-P foil such as elastic modulus, hardness and stiffness determined by tribonano-indenter were tended to increase by addition of $0.1 \mathrm{ppm}$ of thiourea from 77 to $156 \mathrm{GPa}, 6.6$ to $8.9 \mathrm{GPa}$ and 109.6 to $186.6 \mu \mathrm{N} / \mathrm{nm}$, respectively.
\end{abstract}

(Received September 16, 2019; Accepted November 30, 2019)

Keywords: electrodeposition, Ni-P, thiourea

\section{INTRODUCTION}

Electroplated nickel-phosphorus (Ni-P) deposits are utilized in various industries such as automobile components, machinery, aerospace and electronics, because of their good physical, chemical and mechanical properties [1-7].

Usually, the electroless and electrodeposited Ni-P films are classified into three groups according to $\mathrm{P}$ content: high (9 $\mathrm{wt} \%$ above), medium (5 8 $\mathrm{wt} \%)$ or low (1 5 wt\%) P deposits. Previous studies have revealed that the high $\mathrm{P}$ deposits have a fully amorphous-nanocrystalline structure $[5,6,8]$, whereas the low and medium $\mathrm{P}$ deposits can be either crystalline or microcrystalline, with either amorphousnanocrystalline or mixtures of crystalline and amorphous-like structures $[9,10]$.

In terms of properties like hardness and corrosion

- 정무영·남창수: 석사과정, 이병식·최용: 교수

*Corresponding Author: Yong Choi

[Tel: +82-41-550-3537, E-mail: yochoi@dankook.ac.kr]

Copyright (c) The Korean Institute of Metals and Materials resistance, the microhardness of the electroless Ni-P deposit was increased by decreasing $\mathrm{P}$ content $[5,6]$. The Ni-P deposition has a higher dissolution rate than a conventional $\mathrm{Ni}$ deposit. The Ni-P deposit tends to be more uniformly corroded than the conventional $\mathrm{Ni}$ deposits because of its nanocrystalline structure which suffers excessive intergranular corrosion in acid atmosphere [11,12].

These kinds of mechanical and chemical properties of the Ni-P deposits can be controlled by the plating condition such as the temperature of the bath, $\mathrm{pH}$ and the type of additives. Lead acetate is known as a stabilizer in the electroless or electrodeposited Ni-P process. Since the toxic characteristics of the lead have limited the use of this stabilizer, some investigators have chosen other additives such as thiourea $[13,14]$.

It is known that the thiourea enhances the deposition rate by lowering the activation energy of the deposition of the $\mathrm{Ni}$ ion [15]. In case of too much thiourea is adsorbed on the substrate, it can inhibit the deposition reaction by poisoning the catalytic activity of the accidentally formed NiP or metal 
hydroxide nuclei because of the effect of the thiourea on the surface coverage of the substrate [16].

Since the electroless Ni-P process is performed at about $90{ }^{\circ} \mathrm{C}$ and needs more time and energy, the electroplate process is preferred for Ni-P deposit in the field [21]. Although the effect of thiourea on electroless Ni-P deposits have been carried out, little information is available for the effect of thiourea on electroplated Ni-P deposits, especially about mechanical and chemical properties of the Ni-P deposit. Hence, the objective of this study is to investigate the effect of the thiourea on the mechanical and corrosion behavior of the electroplated Ni-P deposits in metallurgical point of view.

\section{EXPERIMENTAL METHODS}

\subsection{Sample preparation}

Ni-P deposits were prepared by conventional electrodeposition method with a modified sulfate bath. Table 1 is the bath of the Ni-P electrodeposition. The Ni-P bath solution is composed of a nickel sulfamate solution, phosphorous acid and boric acid, respectively. Various amounts of thiourea were added to the bath solution over the range of $0.01 \sim 0.1 \mathrm{~g} / \mathrm{L}$ to observe the variation of the properties of the electroplated Ni-P deposits.

The titanium electrode and 304 stainless steel plate as a substrate were used. The substrate was electrolytic polished in $0.2 \mathrm{~N}$ sulfuric acid $\left(\mathrm{H}_{2} \mathrm{SO}_{4}\right)$ to remove debris and prepare a fine surface roughness prior to the electroforming of the $\mathrm{Ni}$ $\mathrm{P}$ deposit. The Ni-P electrodeposition was conducted at $50{ }^{\circ} \mathrm{C}$ and $0.02 \mathrm{~A} / \mathrm{cm}^{2}$ in the modified sulfate bath for an hour to have a similar thickness.

Table 1. Bath composition of the Ni-P electrodeposition

\begin{tabular}{lc}
\hline \multicolumn{1}{c}{ Parameter } & Amount \\
\hline Nickel Sulfamate Solution $[\mathrm{mL}]$ & 300 \\
Phosphorous acid $[\mathrm{g} / \mathrm{L}]$ & 27 \\
Boric acid $[\mathrm{g} / \mathrm{L}]$ & 25 \\
Thiourea $[\mathrm{g} / \mathrm{L}]$ & Controlled \\
\hline
\end{tabular}

2.2 Microstructure, phase analysis and mechanical tests

Surface morphologies and cross sections were observed by atomic force microscopy (Pafm nx II, Em4sys, Korea) and scanning electron microscopy (CX-200TA, Coxem, Korea), respectively. The phase analysis was carried out by X-ray diffractometry (Ultima IV, Rigaku, Japan) at a diffraction angle from $30^{\circ}$ to $100^{\circ}$, step rate of $0.02 \%$ sec and $\mathrm{Cu} \cdot \mathrm{K}_{\alpha}$ line at room temperature. Chemical analysis was performed by energy dispersive spectroscopy (Noran, Thermo, USA). The mechanical properties of the Ni-P deposits prepared with and without thiourea was determined by dynamic nanoindentation method (Ti750, Hysitron, USA) for the specimens with similar thickness.

\section{RESULTS AND DISCUSSION}

\subsection{Chemical analysis}

Figure 1 and Table 2 are the composition variation of the Ni-P deposits with thiourea in the bath. As shown in Fig. 1 and Table 2, the amount of $\mathrm{Ni}$ and $\mathrm{P}$ in the Ni-P deposits were $87.98,96.74,97.47,98.91,98.90$ and $98.41 \mathrm{wt} \%$ and

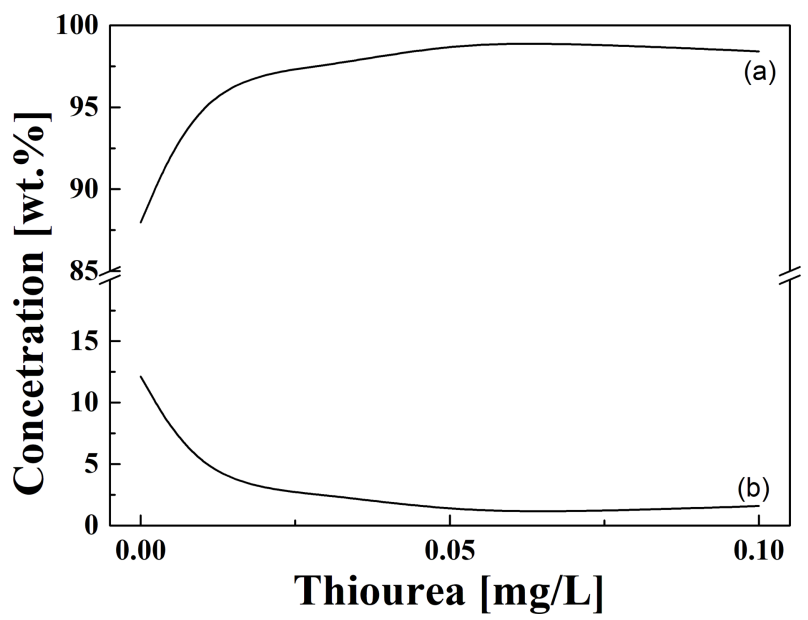

Fig. 1. Ni and $\mathrm{P}$ concentration profiles of the electrodeposited Ni-P layers with thiourea: (a) $\mathrm{Ni}$ (b) $\mathrm{P}$

Table 2. Compositions of the Ni-P deposits with thiourea

\begin{tabular}{ccccccc}
\hline $\mathrm{TU}[\mathrm{g} / \mathrm{L}]$ & 0 & 0.01 & 0.03 & 0.05 & 0.07 & 0.1 \\
\hline $\mathrm{Ni}[\mathrm{wt} \%]$ & 87.98 & 96.74 & 97.47 & 98.91 & 98.90 & 98.41 \\
$\mathrm{P}[\mathrm{wt} \%]$ & 12.12 & 3.36 & 2.53 & 1.19 & 1.10 & 1.59 \\
\hline
\end{tabular}



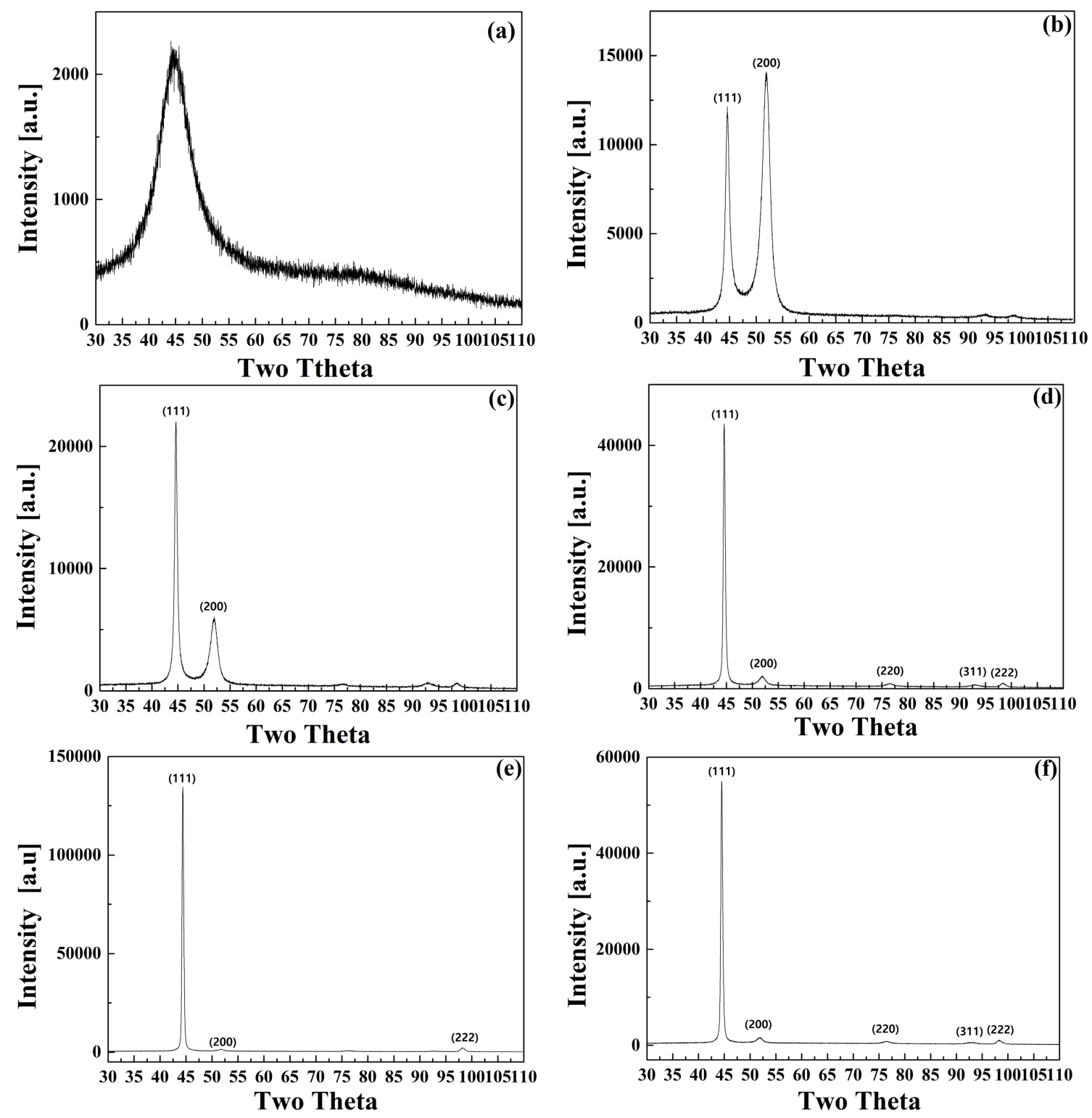

Fig. 2. XRD spectra of the electrodeposited Ni-P layers with thiourea [g/L]: (a) no additives (b) 0.01 (c) 0.03 (d) 0.05 (e) 0.07 (f) 0.1

$12.12,3.36,2.53,1.19,1.10$ and $1.59 \mathrm{wt} \%$, respectively, as the thiourea content changed from 0.01 to $0.1 \mathrm{~g} / \mathrm{L}$. The $\mathrm{P}$ content in the Ni-P deposits decreased increasing the thiourea content in the bath solution whereas the Ni content increased the present data accords with the results reported by Lin et al. [14].

\subsection{Phase identification}

Figure 2 is the XRD spectra of the Ni-P deposits with thiourea. Figure 2-(a) is the spectra of the electrodeposited Ni-P layer without any additives. Figure 2-(b), (c), (d), (e) and (f) are the spectra of the electrodeposited Ni-P layers with thiourea in amounts of $0.01,0.03,0.05,0.07$ and $0.1 \mathrm{~g} / \mathrm{L}$, in order. As shown in Fig. 2, the XRD spectra of the Ni-P deposit without thiourea has a broad peak in the range of $35 \sim 55^{\circ}$, which indicates an amorphous-nanostructure [6,9]. However, the XRD peaks for the deposits with thiourea show the crystalline structure with preferred orientations like splitting into planes of (111), (200), which indicates the crystalline $\mathrm{Ni}$ structure with face centered cubic structure (FCC) [10,20]. Considering these XRD peaks, the Ni-P deposits with thiourea are mainly composed of a $\mathrm{Ni}$ based 
structure. For the $0.01 \mathrm{~g} / \mathrm{L}$ thiourea the intensity of (200) peak was higher than that of the (111) peak. As the amount of thiourea increased in the bath, the (111) peak of the Ni-P deposits increased and (200) peak decreased which dissipates entirely. It supports that the thiourea intensifies the (111) peak and crystallinity of the deposits. Since the amorphousnanostructure is formed for the Ni-P deposit with more than $7 \mathrm{wt} \%$ of $\mathrm{P}[15,17]$, the crystallinity of the Ni-P with thiourea is due to the low content of P of the Ni-P deposits [14].

\subsection{Surface morphologies microstructure and mechanical properties}

Figure 3 is the surface images of the electrodeposited Ni$\mathrm{P}$ deposits with thiourea. As shown in Fig. 3, the surface morphology of the Ni-P deposits without the additive and with $0.01 \mathrm{~g} / \mathrm{L}$ thiourea has almost no nodules and relatively smooth and clean surfaces [18]. However, the surface morphologies of the Ni-P deposits with 0.03, 0.05, 0.07 and $0.1 \mathrm{~g} / \mathrm{L}$ thiourea had more cauliflower-like nodules and subnodules on the surface of the deposits [19]. The surface roughness $\left(\mathrm{R}_{\mathrm{RMS}}\right)$ of the Ni-P deposits determined by atomic force microscopy increased from 6 to $54 \mathrm{~nm}$ with thiourea.

Figure 4 is the cross-sectional view of the Ni-P deposits with thiourea. As shown in Fig. 4, the thickness of the Ni-P deposits with thiourea were 29.6, 18.2, 14.3, 13.5, 12.1 and $10.9 \mu \mathrm{m}$, respectively, which increasing the content of indicates that the thickness of the Ni-P deposits decreases with increasing the content of thiourea in the bath solution for
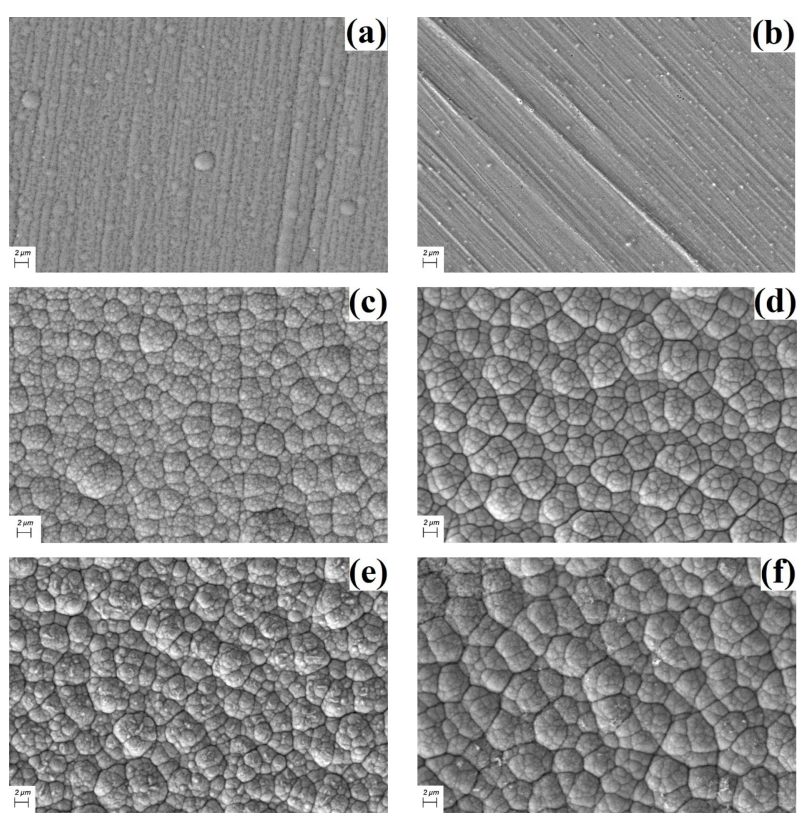

Fig. 3. Surface images of the electrodeposited Ni-P layers with thiourea [g/L]: (a) 0 (b) 0.01 (c) 0.03 (d) 0.05 (e) 0.07 (f) 0.1

the same plating time of one hour. Accordingly, the variation in the surface morphology and the reduction in thickness of the Ni-P deposits with thiourea can be attributed to the effect of thiourea on the grain growth of the nodules. The thiourea adsorbed on the surface of the substrate tends to retard the reduction of the $\mathrm{Ni}$ and $\mathrm{P}$ ions for the nucleation step. Figure 5 is the surface morphologies of the Ni-P deposits. As shown in Fig. 4 and Fig. 5, the size of the columnar grains tends to increase with increasing the content of thiourea.
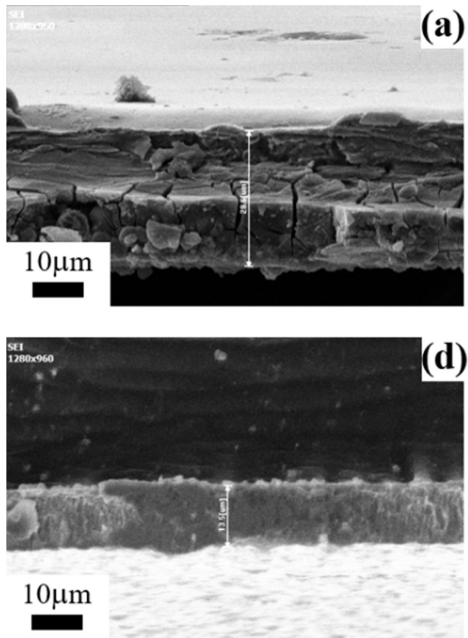
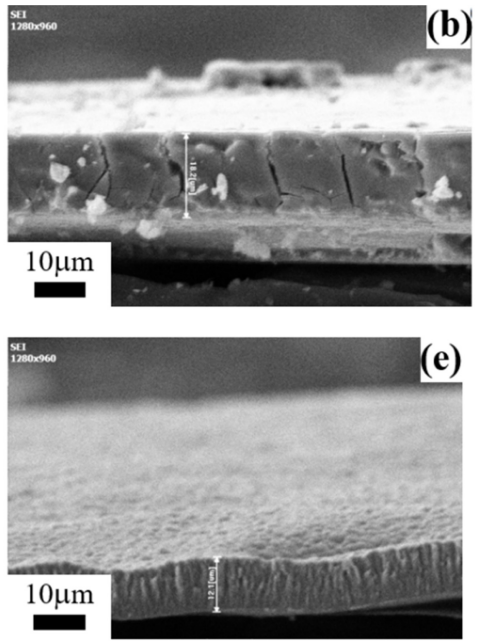

(b)

(e)
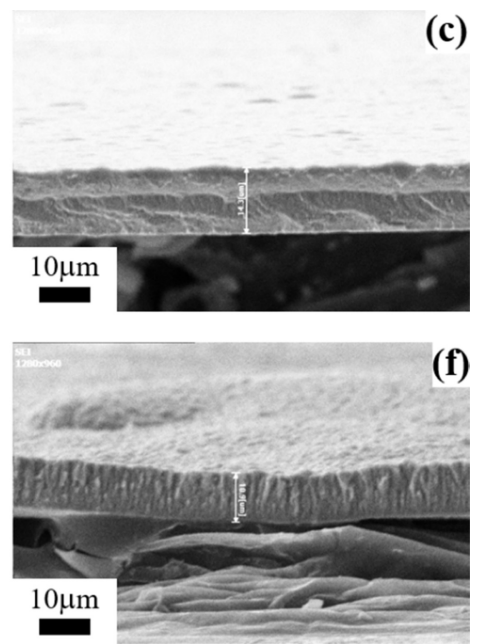

Fig. 4. Cross-sectional view of electrodeposited Ni-P layers with thiourea $[\mathrm{g} / \mathrm{L}]$ : (a) 0 (b) 0.01 (c) 0.03 (d) 0.05 (e) 0.07 (f) 0.1 

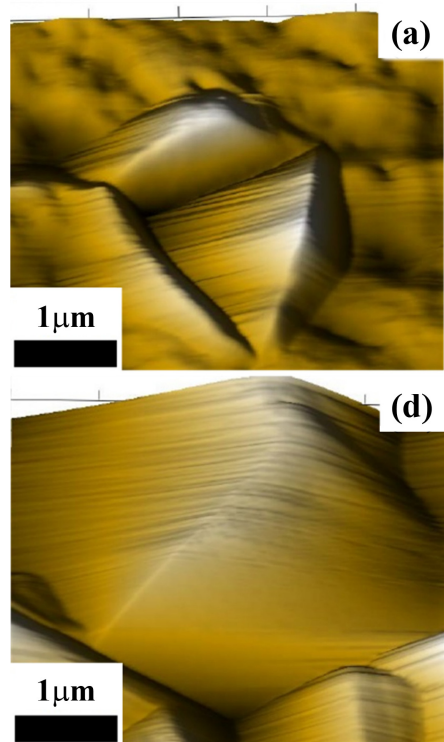

(d)
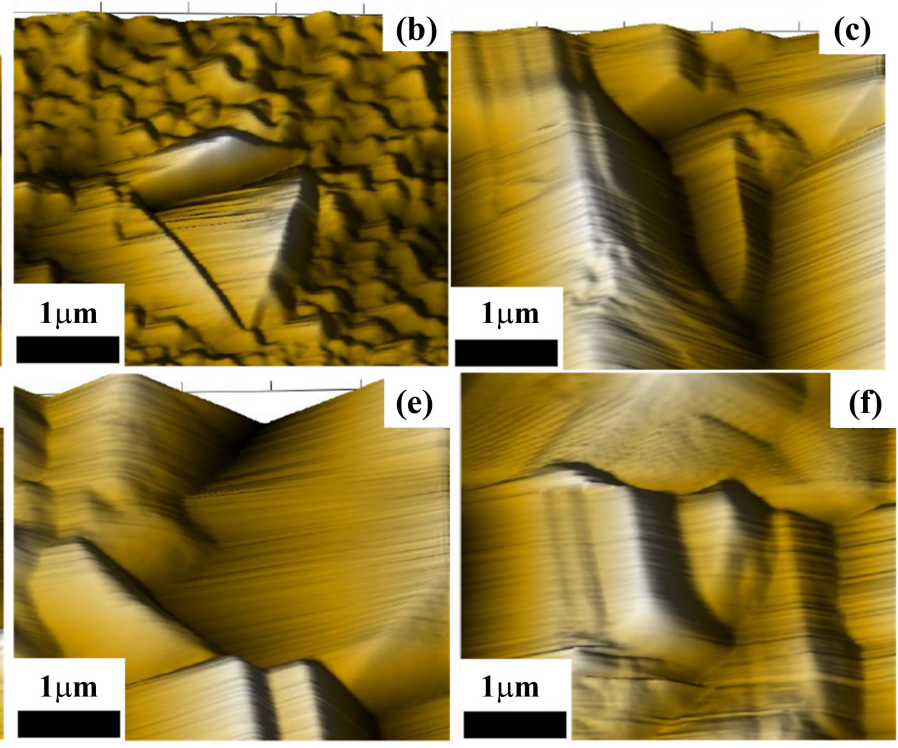

Fig. 5. Surface morphologies of electrodeposited Ni-P layers with thiourea [g/L]: (a) no additive (b) 0.01 (c) 0.03 (d) 0.05 (e) 0.07 (f) 0.1

Table 3. Mechanical properties and surface roughness of the Ni-P deposits with thiourea

\begin{tabular}{ccccc}
\hline Thiourea $[\mathrm{ppm}]$ & $\mathrm{E}_{\mathrm{r}}[\mathrm{GPa}]$ & Hardness $[\mathrm{GPa}]$ & Stiffness $[\mu \mathrm{N} / \mathrm{nm}]$ & $\mathrm{R}_{\mathrm{RMS}}[\mathrm{nm}]$ \\
\hline 0 & $77 \pm 3$ & $6.3 \pm 3$ & $109.6 \pm 3$ & $6 \pm 3$ \\
0.01 & $114 \pm 3$ & $7.7 \pm 3$ & $148.0 \pm 3$ & $11 \pm 3$ \\
0.03 & $116 \pm 3$ & $8.6 \pm 3$ & $142.1 \pm 3$ & $24 \pm 3$ \\
0.05 & $125 \pm 3$ & $8.7 \pm 3$ & $162.2 \pm 3$ & $46 \pm 3$ \\
0.07 & $147 \pm 3$ & $8.9 \pm 3$ & $186.3 \pm 3$ & $50 \pm 3$ \\
0.10 & $156 \pm 3$ & $8.9 \pm 3$ & $18.6 \pm 3$ & $54 \pm 3$ \\
\hline
\end{tabular}

Table 3 shows the surface roughness and mechanical properties of the Ni-P deposits with thiourea. As shown in Table 3, the surface roughness of the deposits increased from 6 to $54 \mathrm{~nm}$ with the addition of $0.1 \mathrm{ppm}$ of thiourea. It implies that the average grain size of the columnar deposits increased with thiourea. The elastic modulus, hardness and stiffness of the Ni-P deposits increased by addition of $0.1 \mathrm{ppm}$ of thiourea, from 77 to $156 \mathrm{GPa}, 6.6$ to $8.9 \mathrm{GPa}$ and 109.6 to $186.6 \mu \mathrm{N} / \mathrm{nm}$, respectively. Since the (111) plane of the deposits preferentially grew in Fig. 2, the preferential growth in crystallinity is one of attributions on the nano-hardening of the Ni-P deposits with thiourea. Besides, the increase in stiffness and elastic modulus related to the decrease in $\mathrm{P}$ contents. According to the non-equilibrium Ni-P binary phase diagram, the Ni-P deposits with a low, medium and high $\mathrm{P}$ content consist of $\beta$ (crystalline), $\beta+\gamma($ crystalline + amorphous $)$ and $\gamma($ amorphous $)$ phases.
Since the $\beta$ phase has greater hardness than the $\gamma$ phase, the nano-hardness of the Ni-P deposits increased as the P content decreased [6].

\section{CONCLUSIONS}

1. The $\mathrm{P}$ content of the Ni-P deposits decreased with increasing thiourea content in the bath, whereas, the $\mathrm{Ni}$ content of the deposits increased with thiourea.

2. The thiourea intensified the (111) peak and crystallinity of the electrodeposited Ni-P deposits.

3. The surface morphologies of deposits without additive and with $0.01 \mathrm{~g} / \mathrm{L}$ of thiourea had almost no nodules and were relatively smooth and clean. Those of the Ni-P deposits with $0.03,0.05,0.07$ and $0.1 \mathrm{~g} / \mathrm{L}$ thiourea had more cauliflower-like nodules and sub-nodules on the surface. The surface roughness of the Ni-P deposits determined by atomic 
force microscopy increased from 6 to $54 \mathrm{~nm}$ with the addition of $0.1 \mathrm{ppm}$ of thiourea.

4. The elastic modulus, hardness and stiffness of the Ni-P deposits with thiourea increased from 77 to $156 \mathrm{GPa}, 6.6$ to 8.9 $\mathrm{GPa}$ and 109.6 to $186.6 \mu \mathrm{N} / \mathrm{nm}$, respectively. The hardening of the Ni-P deposits with thiourea is related to the preferential growth of crystallinity and $\mathrm{P}$ content. Increased stiffness and elastic modulus resulted from a decrease in $\mathrm{P}$ content which led to the $\beta$ phase instead of $\gamma$ phase.

\section{ACKNOWLEDGEMENT}

C. S. Nam and B. S. Lee thank the National Research Foundation of Korea (NRF), granted financial resource from the Ministry of Science, ICT and Future Planning (No. 2016M2B2B1945084), Republic of Korea.

\section{REFERENCES}

1. P. Peeters, G. V. D. Hoorn, T. Daenen, A. Kurowski, and G. Staikov, Electrochim. Acta. 47, 161 (2001).

2. R. Narayan and M. N. Mungole, Surf. Technol. 24, 233 (1985).

3. A. P. Ordine, S. L. Diaz, I. C. P. Margarit, O. E. Barcia, and O. R. Mattos, Electrochim. Acta. 51, 1480 (2006).

4. A. A. Ashtiani, S. Faraji, S. A. Iranagh, and A. H. Faraji, Arabian J. Chem. 10, 1541 (2017).

5. R. Taheri, Ph. D. Thesis, pp.23-24, University of Saskatchewan, Saskatoon (2002).

6. S. R. Anvari, S. M. Monirvaghefi, and M. H. Enayati, Surf Eng. 31, 693 (2015).
7. H. B. Lee, D. S. Wuu, C. Y. Lee, and C. S. Lin, Tribology Int. 43, 235 (2010).

8. M. Czagany, P. Baumli, and G. Kaptay, Appl. Surf. Sci. 423, 160 (2017).

9. A. Hadipour, S. M.Monirvaghefi, and M. E. Bahrololoom, Surf. Eng. 31, 399 (2015).

10. I. Baskaran, T. S. N. Sankara Narayanan, and A. Stephen, Mater. Chem. Phys. 99, 117 (2006).

11. R. Rofagha, U. Erb, D. Ostrander, G. Palumbo, and K. T. Aust, Nanostruct. Mater. 2, 1 (1993).

12. R. Rofagha, R. Langer, A. M. El-Sherik, U. Erb, G. Palumbo, and K. T. Aust, Scripta Metall. Mater. 25, 2867 (1991).

13. A. R. Rahimi, H. Modarres, and M. Abdouss, Surf. Eng. 25, 367 (2009).

14. K.-L. Lin and J.-W. Hwang, Mater. Chem. Phys. 76, 204 (2002).

15. G. O. Mallory and J. B. Hajdu, Electroless Plating: Fundamentals and Applications, AESF, Orlando, FL, pp.118-124 (1990).

16. M. Saitou, Int. J. Electrochem. Sci. 11, 1651 (2016).

17. B. Ke, J. J. Hoekstra, B. C. Sison, Jr., and D. Trivich, J. Electrochem. Soc. 106, 382 (1959).

18. U. S. Mohanty, B. C. Tripathy, S. C. Das, and V. N. Misra, Metall. Mater. Trans. B 36, 735 (2005).

19. N. Parvini-Ahmadi and M. A. Khosravipour, Int. J. ISSI. 1, 31 (2004).

20. M. S. Kim, Y. J. Kim, and Y. H. Kim, Korean J. Met. Mater. 56, 652 (2018).

21. Y. Huang, X. Peng, Y. Yang, H. Wu, X. Sun, and X. Han, Met. Mater. Int. 24, 1172 (2018). 